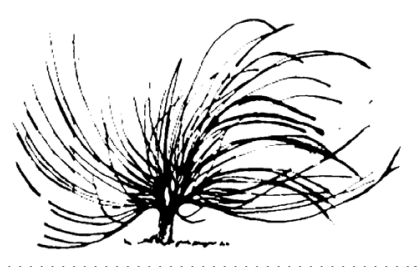

\title{
El portafolio, la bitácora y el dictado: estrategias didácticas para mejorar el proceso de redacción
}

\author{
María Eugenia Ugalde Villalobos ${ }^{1}$ \\ Universidad Nacional \\ Costa Rica \\ maria.ugalde.villalobos@una.cr
}

\begin{abstract}
Resumen
Este ensayo presenta estrategias para mejorar el proceso de redacción como el uso de las bitácoras, el portafolio y el dictado con la combinación de la metodología semipresencial o bimodal en el curso de Comunicación y redacción de la Escuela de Secretariado Profesional de la Universidad Nacional. Además, se enfatiza en que los signos de puntuación y la ortografía mejoran mucho cuando el estudiantado pone en práctica las herramientas antes mencionadas ya que son los contenidos que más fallan en el proceso de redacción. Como conclusión, se considera que la clave para el mejoramiento de la redacción es la realimentación que se da entre docente - estudiantado, clases presenciales - clases virtuales y revisión - corrección.
\end{abstract}

Palabras claves: bimodalidad, bitácora, dictado, estrategias didácticas, portafolio, redacción.

\begin{abstract}
This essay presents strategies to improve the writing process with the use of log books, porftolios, and dictation
\end{abstract}

Recibido: 23 de julio de 2019. Aprobado: 29 de abril de 2020.

http://dx.doi.org/10.15359/rep.15-2.2

1 Máster en Educación con Énfasis en Docencia Universitaria. Licenciatura en Literatura y Lingüística con énfasis en Literatura. Bachiller en Filología Española. Académica de la Escuela de Secretaria Profesional, Universidad Nacional de Costa Rica. https://orcid.org/0000-0002-1635-1193 
combined with a hybrid or blended-learning methodology in the course of Communication and Writing of the School of Professional Secretariat at Universidad Nacional. In addition, it emphasizes that punctuation and spelling improve greatly when students put into practice the aforementioned tools since those are the contents that students fail the most in the writing process. In conclusion, the key to improving writing is the feedback given among the teacher - students, face-to-face classes - virtual classes and revision - correction.

Keywords: log books, dictation, teaching strategies, portfolio, writing, blended learning

\section{Introducción}

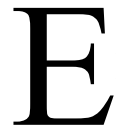

1 curso Comunicación y redacción pertenece a las carreras de Educación Comercial y Administración de Oficinas de la Escuela de Secretariado de la Universidad Nacional de Costa Rica y se desarrolla en el segundo nivel de ambas carreras. A pesar de que los estudiantes ya han cursado Técnicas de redacción, llegan con serias dificultades en los temas de puntuación y ortografía en ese nivel. Por lo anterior, la autora trató de buscar diferentes estrategias para que los estudiantes pusieran en práctica el proceso de redacción, en el cual también estuviera implícita la realimentación de los temas mencionados.

Por eso, el objetivo de este ensayo es describir las experiencias adquiridas en el curso Comunicación y redacción con el desarrollo del portafolio, las bitácoras y el dictado como herramientas de evaluación para mejorar la redacción. También, en este trabajo se abarcarán los conceptos de cada técnica, la metodología utilizada y los resultados obtenidos.

Según Müller (2013), "redactar significa expresar por escrito pensamientos, conocimientos o hechos que se hayan producido en la realidad, sin que entren en juego ni la imaginación ni la creatividad del escritor" (p. 6). Redactar es un proceso complicado para el estudiantado en diferentes niveles: escolar, secundaria y universitario, ya que supone el conocimiento de temas como los vicios de dicción, la acentuación y los signos de puntuación; estos últimos son los contenidos que los estudiantes más fallan en el proceso de redacción.

Por ello, en este escrito, se desarrollará el uso de los instrumentos mencionados, específicamente, en el nivel universitario como forma de evaluación en el curso de redacción. Sin embargo, la metodología y 
la evaluación desarrolladas se pueden utilizar en la escuela primaria y centros de enseñanza de secundaria.

De acuerdo con la Ortografía de la Lengua Española (2014),

Los signos de puntuación son los signos ortográficos que organizan el discurso para facilitar la comprensión, poniendo de manifiesto las relaciones sintácticas y lógicas entre sus diversos constituyentes, evitando posibles ambigüedades y señalando el carácter especial de determinados fragmentos (citas, incisos, intervenciones de distintos interlocutores en un diálogo, etc.). De la puntuación depende en gran medida la comprensión cabal de los textos escritos, de ahí que las normas que la regulan constituyan un aspecto básico de la ortografía. (p. 282)

Según lo anterior y la experiencia acumulada a través de los años, en el curso de Comunicación y redacción, el estudiantado ingresa a la universidad con serios problemas de redacción, a pesar de que es un contenido que se estudia en la educación secundaria cinco años y seis en la primaria. Debido al grado de deficiencia que se presenta en el nivel universitario, en la aplicación de la ortografía y la puntuación, el uso de la bitácora como instrumento de evaluación en el proceso de redacción llamó la atención para que el estudiantado tenga una práctica constante. Por ejemplo, algunos estudiantes suelen tildar muchas palabras que terminan en on ya que desde la escuela aprenden la incorrecta regla de que todo lo que termina en on se tilda. La autora también fue docente en secundaria y le llamó mucho la atención esa regla y un día les preguntó a los estudiantes de sétimo año: por qué tildan todo lo que termina en on, ellos contestaron que así les enseñaron en la escuela, por lo tanto, es un error que se trae desde la educación primaria y llega a la universitaria. Dicha deficiencia se debe eliminar, ya que el estudiantado del curso Comunicación y redacción, serán los futuros profesionales de la educación secundaria en el área de educación comercial, quienes, a su vez, se convertirán en formadores que deben dominar el tema de la redacción y, por ende, la ortografía y puntuación. Además, los profesionales en Administración de Oficinas serán el personal de diversas instituciones públicas y privadas que deberán también dominar los temas antes mencionados. 
La metodología que se desarrolló en el curso fue la semipresencial o bimodal, con la plataforma Aula Virtual de la Universidad Nacional, la cual se combinó con el uso de las bitácoras, el portafolio y los dictados. Cada uno de ellos, se amplía en los siguientes apartados.

\section{Bimodalidad y pedagogía}

Con el fin de que haya una mayor realimentación en el aprendizaje y enseñanza del curso Comunicación y redacción, se emplea la metodología b-learning, la cual es un proceso semipresencial en donde se utilizan recursos virtuales y físicos en un curso que incluye clases presenciales, así como actividades e-learning (aprendizaje electrónico) que toma las ventajas de las dos modalidades.

La Universidad Nacional cuenta con la plataforma Aula Virtual, la cual fue aprovechada por la autora para desarrollar el curso de forma bimodal, las herramientas que más utiliza dentro de esta plataforma son los foros y las tareas; el portafolio y las bitácoras se trabajan no solo en las clases presenciales sino también en las virtuales.

Por lo anterior, el curso se desarrolló de forma bimodal porque los estudiantes reciben $40 \%$ de las clases en forma virtual y $60 \%$ presencial, en otras palabras, siete clases virtuales y diez presenciales.

En las clases presenciales se abarcaron los contenidos como vicios de dicción, el párrafo, tipos de oraciones, uso de conectores, el escrito y se realizaron las respectivas prácticas y, en las clases virtuales, los estudiantes redactaron producciones textuales descriptivas, narrativas, expositivas y argumentativas en las que aplicaron todo lo visto de forma presencial y en cursos anteriores, como la ortografía y los signos de puntuación.

Este tipo de modalidad facilita la pedagogía de la redacción, ya que se trabaja $60 \%$ presencial y $40 \%$ virtual. Esta última es una buena técnica porque el estudiantado tiene más tiempo para redactar sus producciones textuales y el docente también cuenta con más tiempo para corregir las redacciones. Además, se presenta la autoevaluación cuando cada estudiante revisa sus propios trabajos y la heteroevaluación se da cuando el docente es el que revisa el documento escrito por este, es decir, se da un proceso de realimentación.

\section{El uso de la bitácora}

Según Calderón (2007), la bitácora funciona para mejorar el proceso de enseñanza y aprendizaje cuando se han realizado los registros 
correspondientes y se ha revisado detenidamente lo que sucede en el aula. De esa manera, el docente puede decidir qué aspectos puede transformar.

En el caso del curso de Redacción, los estudiantes redactan cinco bitácoras de lo que acontece en la clase, la docente las revisa y señala los errores, pero no los corrige, con el fin de que el estudiantado haga una segunda versión y determine sus propios errores.

Cada estudiante debe buscar el tipo de error y él mismo recibe su propia realimentación cuando corrige el texto. Esta se presenta mediante las dualidades docente-estudiantado, revisión-corrección y clases presenciales-virtuales; es decir, con la revisión y corrección constantes de las bitácoras, el estudiante realiza una sistematización de los errores más evidentes con el fin poder disminuirlos en las próximas redacciones. Cabe destacar que el proceso de redacción mejora siempre y cuando haya un intercambio de aprendizaje entre el docente y el educando con el fin de que haya modificaciones para corregir sus faltas, es decir, devoluciones constantes de los trabajos.

De acuerdo con el Programa Intel para Educar (s. f.)

La realimentación mejora el trabajo del estudiante, al resaltar el progreso en lugar de la deficiencia. Con la realimentación del progreso, un estudiante será capaz de automonitorearse satisfactoriamente, así como de tener mayores aspiraciones para futuros trabajos, una mayor autosuficiencia y un mejor desempeño general. Al tomarse el tiempo para sentarse con un estudiante y ofrecerle una crítica constructiva, la ayuda necesaria, sugerencias y una realimentación positiva, los docentes pueden impactar de manera positiva el aprendizaje del estudiante. (p. 1)

La práctica constante durante el desarrollo del curso es primordial para que los estudiantes reciban realimentación, la cual es una gran estrategia de aprendizaje en el proceso de redacción porque ellos aprenden de sus propios errores.

En el mismo salón de clases, se practica la dualidad docente-estudiante cuando este redacta un texto de diez a quince líneas y la docente lo corrige junto con el estudiante para que le explique cada falla y cómo modificarlo; en un texto corto, hay fallas, las cuales son insumos para la realimentación y el aprendizaje. 
Según las redacciones de las bitácoras, el error que se presenta con mayor frecuencia es el uso incorrecto de la coma a pesar de que su uso se estudia y se realizan ejercicios de aplicación durante las clases. El error que se refleja con mayor frecuencia es la colocación de la coma entre el sujeto y el verbo, lo cual es incorrecto, por lo tanto, en la próxima redacción ese error ya no se cometerá porque se ha hecho una realimentación del tema y práctica constante.

Los signos de puntuación es un tema que se estudia desde la edad escolar, seis años y, en la secundaria, cinco años; sin embargo, los estudiantes llegan con grandes lagunas en esos temas al nivel universitario, como lo señalan Rodino y Ross (1985) según se citan en Sánchez (2006), “después de varios años de instrucción formal, los estudiantes costarricenses llegan a las universidades con serias deficiencias en cuanto al manejo del registro escrito" (p. 1).

La experiencia de la autora indica que el hecho de que haya una práctica constante con la redacción y corrección de las bitácoras mejora la aplicación de los signos de puntuación y la ortografía de los estudiantes universitarios; es decir, el estudiantado debe producir sus propios escritos con el fin de que el mismo los revise y corrija; posteriormente, sin dejar de lado las observaciones de la persona docente, quien da la corrección final a los textos. Luego, el estudiante debe realizar una comparación de los errores cometidos para que tenga un insumo para su propio aprendizaje.

En cuanto a la dualidad virtual-presencial, el estudiante redacta su bitácora a mano como tarea, la docente la revisa y señala el error, pero no lo corrige, el discente es quien se encarga de esa labor. Además, las correcciones de las bitácoras se entregan de forma virtual, ya que la primera versión se entrega de forma física. De esa manera, también se crea un portafolio virtual. Además, se da la flexibilidad temporal porque el estudiante cuenta con un poco más de tiempo para redactar, revisar, corregir y entregar sus trabajos.

Según Calderón (2007), la bitácora funciona para mejorar el proceso de enseñanza y aprendizaje, dicha teoría se cumple en el curso de Comunicación y redacción porque el objetivo es realizar un registro de los errores más evidentes, con el fin de que se fomente la mejoría en el proceso de redacción en las próximas bitácoras, situación que sucede con esos escritos. 


\section{El portafolio}

El portafolio es otro instrumento de evaluación muy eficaz tanto para la docente como para la persona estudiante ya que es una estrategia de aprendizaje muy productiva para que haya un cambio en el proceso de enseñanza y aprendizaje de la redacción.

La intención de que se elabore el portafolio es que el estudiantado realice sus propias producciones textuales en las que utilice los signos de puntuación y el mismo monitoree su propio progreso, es decir, se dé un proceso de autoevaluación.

Para Guerrero (2015), el portafolio es una estrategia didáctica para enriquecer la práctica pedagógica. Además, esta se aprovecha para la investigación en el aula, la reflexión pedagógica, el ejercicio docente, la autonomía, la universidad y su desarrollo y el estudiante avanza en su desarrollo profesional con la mediación del profesor.

El portafolio en este curso se desarrolla mediante la evaluación de dos redacciones a mano con una extensión de dos páginas, con un valor de $10 \%: 5 \%$ el texto original y $5 \%$ las correcciones de esas redacciones. La evaluación de esas redacciones implica la corrección del acento, las mayúsculas, la ortografía, la puntuación, preposiciones, vicios de dicción y la estructura. En la primera revisión, la docente se encarga de buscar los errores y los señala; en la corrección, el estudiante busca el tipo de error y los corrige. En la Tabla 1, se muestra la escala de calificación para corregir las producciones textuales.

\section{Tabla 1}

Escala para calificar la producción de texto (portafolio)

\begin{tabular}{|l|l|l|l|}
\hline \multicolumn{1}{|c|}{ Indicadores } & $\begin{array}{c}\text { 3 pts. } \\
\text { No hay } \\
\text { errores }\end{array}$ & $\begin{array}{c}\mathbf{2} \text { pts. } \\
\text { De uno } \\
\text { a tres } \\
\text { errores }\end{array}$ & $\begin{array}{c}\mathbf{1} \text { pto. Más } \\
\text { de tres } \\
\text { errores }\end{array}$ \\
\hline $\begin{array}{l}\text { 1. Empleó la tilde de acuerdo con las reglas } \\
\text { de acentuación en palabras agudas, graves, } \\
\text { esdrújulas y sobreesdrújulas y el uso de la } \\
\text { tilde diacrítica. }\end{array}$ & & & \\
\hline $\begin{array}{l}\text { 2. Empleó la letra mayúscula de acuerdo } \\
\text { con la normativa. }\end{array}$ & & & \\
\hline $\begin{array}{l}\text { 3. Aplicó los principios ortográficos en el } \\
\text { uso de las letras. }\end{array}$ & & & \\
\hline
\end{tabular}




\begin{tabular}{|c|c|c|c|}
\hline Indicadores & $\begin{array}{l}3 \text { pts. } \\
\text { No hay } \\
\text { errores }\end{array}$ & $\begin{array}{l}2 \text { pts. } \\
\text { De uno } \\
\text { a tres } \\
\text { errores }\end{array}$ & $\begin{array}{l}1 \text { pto. Más } \\
\text { de tres } \\
\text { errores }\end{array}$ \\
\hline $\begin{array}{l}\text { 4. Empleó los diferentes signos de } \\
\text { puntuación de acuerdo con la normativa. } \\
\text { Empleó correctamente la sangría. }\end{array}$ & & & \\
\hline $\begin{array}{l}\text { 5. Evitó ambigüedades en el escrito, } \\
\text { la repetición de palabras e ideas, la } \\
\text { redundancia y el mismismo, el cosismo y } \\
\text { los verbos comodines. }\end{array}$ & & & \\
\hline $\begin{array}{l}\text { 6. Empleó las preposiciones y los } \\
\text { marcadores textuales. Evitó el queísmo, } \\
\text { dequeísmo, entre otros. }\end{array}$ & & & \\
\hline $\begin{array}{l}\text { 7. Construyó estructuras gramaticales } \\
\text { correctas: aplicó las normas morfológicas } \\
\text { y sintácticas, el uso del gerundio, la } \\
\text { concordancia entre el pronombre y su } \\
\text { antecedente, entre los elementos del grupo } \\
\text { nominal y sus modificadores, entre el } \\
\text { sujeto y el verbo. }\end{array}$ & & & \\
\hline 8. Uso de la tercera persona & & & \\
\hline $\begin{array}{l}\text { 9. Realizó la lluvia de ideas para la } \\
\text { redacción ( } \mathbf{1} \text { punto) y cumplió con las } \\
\text { instrucciones dadas ( } \mathbf{1} \text { punto) }\end{array}$ & & & \\
\hline $\begin{array}{l}\text { Estructura } \\
\text { 10. Redactó la introducción ( } 1 \text { punto), el } \\
\text { desarrollo ( } 2 \text { puntos) y la conclusión ( } 1 \\
\text { punto) según la estructura y la función } \\
\text { requerida de cada una de estas partes dentro } \\
\text { del escrito. }\end{array}$ & & & \\
\hline $\begin{array}{l}\text { 11. Enlazó las ideas de forma coherente } \\
(2 \text { puntos). Todos los párrafos aparecen } \\
\text { concatenados en forma lógica y secuencial } \\
(2 \text { puntos). Planteó argumentos críticos que } \\
\text { respalden su posición ( } 2 \text { puntos). }\end{array}$ & & & \\
\hline
\end{tabular}

Nota: Programa del curso Comunicación y redacción.

Sánchez (2006), plantea con respecto a un curso de redacción que se llevó a cabo en la Universidad de Costa Rica: 
La metodología consistió en la redacción de un texto por semana; la revisión de este por parte del profesor, quien procedió a marcar las faltas o aspectos por mejorar mediante un sistema de claves; la explicación de aspectos teóricos en clase y el análisis de textos de diverso tipo para ejemplificar las estrategias estudiadas y la resolución de ejercicios en clase y como tarea. Los estudiantes tuvieron que llevar un fólder en el que guardaban todos los borradores y las redacciones corregidas en orden, con el fin de que ellos mismos pudieran apreciar el progreso. También se les pidió que rehicieran algunas de sus redacciones, después de que el profesor les entregara la primera versión con las observaciones y las claves. (p. 72)

Al realizar una comparación de la metodología utilizada en el curso de Comunicación y redacción y la planteada por Sánchez (2006), se concluye lo siguiente: la redacción de un texto por semana es la bitácora que cada estudiante escribe en el curso que plantea la autora de este ensayo, el hecho de que los alumnos llevaran un folder para guardar los borradores hace referencia al portafolio y rehacer algunas de las redacciones son las correcciones de las bitácoras realizadas por los estudiantes, es decir, las correcciones que forman parte del portafolio. En este caso, se utilizan los nombres apropiados desde el punto de vista pedagógico en el curso de Comunicación y redacción.

En el curso de redacción, el portafolio se utiliza con el fin de que el estudiantado realice el ejercicio de guardar cada una de las bitácoras y redacciones con las respectivas correcciones que elaboró, con el fin de que observe el proceso de avance en su aprendizaje.

Además, si se compara lo planteado por Guerrero (2015) en cuanto a que el estudiante avanza en su desarrollo profesional con la mediación del docente, es una teoría que se comprueba en el curso Comunicación y redacción porque se presenta la dualidad profesor-estudiante cuando se da el intercambio de las revisiones de la producción de textos entre la docente y el estudiantado, ya que en la corrección de cada escrito se presenta la realimentación, la cual contribuye en el proceso de enseñanza y aprendizaje. Según el proceso de evaluación y autoevaluación, los errores que más sobresalen son el uso incorrecto del acento y las repeticiones. Con la puesta en práctica de las bitácoras, el estudiantado mejora en gran medida la ortografía, entonces cuando presenta el portafolio ese indicador es uno de los mejor puntuados. 


\section{El dictado}

Otra estrategia que se utiliza para el aprendizaje y enseñanza de la redacción es el dictado con el fin de que el estudiantado ejercite las dificultades ortográficas estudiadas. Para Fis y González (2015), existen varios tipos; sin embargo, el que compete a este ensayo, es el dictado de control, el cual tiene como objetivo evaluar el trabajo. Debe realizarse con cierta periodicidad para conocer en qué medida se dominan los contenidos ortográficos estudiados, para tomar las medidas en los casos necesarios.

Este tipo de dictado se relaciona con el que se practica en la clase de redacción porque se realiza con el fin de saber cuáles letras son las que presentan mayor dificultad para que el estudiantado realimente sus conocimientos con la ayuda del profesorado.

Cuando se desarrolla la clase, se dicta un texto, no palabras, porque en el curso anterior, se dictan de acuerdo con la letra que se vaya estudiando, por ejemplo, $b, v, c, s, z, h$ y la acentuación. Si el dictado es de un texto, se comprueba si el estudiante aplica bien las letras, por ejemplo, el uso de ha y $a$, ya que ese tipo de grafía se puede comprobar en el contexto, así como los parónimos u homónimos.

De acuerdo con los dictados realizados, la acentuación es el error ortográfico en el que más fallan los estudiantes y el uso de la $h$, sobre todo cuando se refiere al uso de la $h a$ y la $a$. Ha se aplica cuando se escribe el participio ado, ido, to, so, cho y la $a$ cuando se alude a una preposición.

Por otra parte, con los dictados también se da esa cadena de realimentación porque el docente dicta el texto, lo corrige y el estudiante debe revisar los aspectos como las mayúsculas, la acentuación y la ortografía para que en los próximos no cometa los mismos errores y haya una mejora en el proceso de escritura.

A muchos estudiantes, les cuesta reconocer el acento prosódico del átono, por eso no saben dónde colocar la tilde, cuya función es "señalar que la sílaba de la que forma parte la vocal sobre la que se escribe es la que porta el acento prosódico de la palabra" (Real Academia Española, 2014, p. 230).

Según la Ortografía de la Real Academia Española (2014), "el acento es la diferencia en la pronunciación de una determinada sílaba, que establece un contraste entre ella y el resto de las que la integran" (p. 190).

Desde este punto de vista, con este tipo de enseñanza se construye pedagogía porque es una disciplina que se convierte en un arte 
cuando el docente aplica sus saberes para crear y recrear formas de enseñanza de la redacción, sabe hacer bien su trabajo y tiene capacidad profesional en los diversos contextos, por ejemplo, cuando se trabaja en zonas rurales con la escasez de recursos y en zonas urbanas donde abundan los recursos pedagógicos. La docente debe buscar su propia metodología para conseguir que el estudiante mejore la acentuación y el dictado es una buena técnica.

Según Gómez y Mora (2011), la pedagogía es una disciplina social y práctica para la vida y no solo para el trabajo. En el caso de los aprendizajes de los que se hablan en este ensayo, son conocimientos que se aprenden para la vida, porque el saber ortografía, puntuación, acentuación y redactar proporciona una buena imagen social y profesional; por el contrario, al no dominarlos, la misma sociedad se encarga de reprimir a las personas que presentan esos errores.

\section{Conclusiones}

Las bitácoras son una excelente herramienta de realimentación por las revisiones que realiza la persona docente para verificar el nivel de progreso del estudiante. Asimismo, tanto uno como el otro monitorean su aprendizaje en el proceso de redacción, sobre todo cuando aplica los signos de puntuación y la ortografía.

El portafolio funciona como un ejercicio docente con el fin de que el estudiantado almacene las bitácoras y reflexione pedagógicamente acerca de sus propios errores. Además, adquiere autonomía para convertirse en autoevaluador de sus producciones textuales y puede corregir errores, como la puntuación, las repeticiones, la ortografía, los vicios de dicción y la acentuación.

Con la metodología que se utilizó en el curso de Comunicación y redacción a lo largo de varios ciclos lectivos, se han percibido cambios como la mejora en la puntuación que es el rubro en que más falla el estudiantado a la hora de redactar.

El dictado es una excelente técnica para afianzar hábitos, habilidades y destrezas en la enseñanza y aprendizaje de la ortografía. Además, es una forma para corregirse a sí mismo cuando se escribe determinada palabra de acuerdo con su contexto.

La bitácora, el portafolio y el dictado utilizados en el desarrollo de este ensayo llamaron la atención porque se presentan dualidades en las que tanto el estudiantado como la docente reciben realimentación, 
la cual es fundamental para la práctica constante en el proceso de enseñanza y aprendizaje de la redacción.

Además, la construcción del conocimiento pedagógico del proceso de redacción se construye mediante la realimentación con críticas constructivas y sugerencias positivas constantes entre la docente y el estudiantado no solo de forma presencial sino también virtual, metodología que funciona muy bien en el proceso de enseñanza y aprendizaje de la redacción.

Como recomendación, se sugiere seguir las estrategias planteadas en este ensayo con el fin de que haya un mayor aprendizaje en el proceso de redacción, ya que para el estudiantado la realimentación es elemental para que aprenda de sus propios errores.

\section{Referencias}

Calderón, G (2007). Trascender la bitácora. Revista Electrónica Sinéctica, 29, 74-78. Recuperado de https://www.redalyc.org/articulo. oa? $\mathrm{id}=99815739011$

Gómez-Torres, J. R. (2016). Pedagogía, una disciplina indisciplinada: Límites de un debate y sus perspectivas ético políticas. Revista Educare, 20(3), 1-12. Recuperado de https://www.revistas.una.ac. cr/index.php/EDUCARE/article/download/7063/9906?inline=1

Guerrero, H. (2015). El portafolio: una herramienta facilitadora del cambio en la educación superior desde la práctica didáctica. Zona Próxima, 22, 143-145. Recuperado de https://www.redalyc.org/ articulo.oa? id $=85339658011$

Fis, O. y González, E. (2015). Consideraciones teóricas y metodológicas relacionadas con el tratamiento del dictado en la enseñanza de la ortografía. Revista Educación y Sociedad, 13(2), 50-59. Recuperado de http://revistas.unica.cu/index.php/edusoc/article/ view/128/pdf

Müller, M. (2013). Curso básico de redacción. San José, Costa Rica: EUCR.

Programa Intel para Educar. (s. f.). Realimentación de docente y compañeros. Recuperado de https://www.intel.la/content/dam/www/ program/education/lar/xl/es/images/instructional-strategies-feedback.pdf

Real Academia Española. (2014). Diccionario de la Lengua Española $\left(22^{\mathrm{a}}\right.$ ed.). Madrid. España. 
Sánchez, C. (2006). ¿Cuestión de Método? Sobre los cursos remediales universitarios de expresión escrita. Revista de Educación, 30(1), 65-81. Recuperado de https://revistas.ucr.ac.cr/index.php/ educacion/article/view/1795 
